\title{
Ultrafast $x$-ray imaging of pulsed plasmas in water
}

\author{
Christopher Campbell $\odot,{ }^{1}$ Xin Tang $\odot,{ }^{1}$ Yancey Sechrest $\odot,{ }^{2}$ Kamel Fezzaa $\odot,{ }^{3}$ Zhehui Wang $\odot,{ }^{2, *}$ and David Staack ${ }^{1, \dagger}$ \\ ${ }^{1}$ Department of Mechanical Engineering, Texas A\&M University, College Station, Texas 77843, USA \\ ${ }^{2}$ Los Alamos National Laboratory, Los Alamos, New Mexico 87545, USA \\ ${ }^{3}$ X-ray Science Division, Advanced Photon Source, Argonne National Laboratory, Argonne, Illinois 60439, USA
}

(Received 12 February 2020; revised 15 July 2020; accepted 15 March 2021; published 8 June 2021)

\begin{abstract}
Ultrafast x-ray imaging is useful for diagnosing a wide range of nanosecond-time scale physical processes, particularly those concealed by optical emission or otherwise opaque objects. Here we use the ultrafast x-ray imaging facilities at Argonne National Laboratory's Advanced Photon Source to interrogate nanosecond-pulsed single-electrode plasma initiation processes in water $(+25 \mathrm{kV}, 10 \mathrm{~ns}, 5 \mathrm{~mJ})$, with supporting nanosecond optical imaging and $\mathrm{x}$-ray diffraction computational model. These results clearly resolve narrow $(\sim 10 \mu \mathrm{m})$ lowdensity plasma channels during initiation time scales typically obscured by optical emission. This multiphase environment is not well described in literature, and these experimental results appear to be inconsistent with prevailing breakdown initiation hypotheses. This work also proposes this plasma process as a cheap, compact, and repeatable benchmark imaging target with hypersonic phenomena $(29.1 \mathrm{~km} / \mathrm{s})$ and very high-power densities $\left(1 \mathrm{TW} / \mathrm{cm}^{2}\right)$, useful for the development of next-generation ultrafast imaging of inertial confinement fusion and other nanosecond processes of interest.
\end{abstract}

DOI: 10.1103/PhysRevResearch.3.L022021

\section{INTRODUCTION}

Nanosecond-pulsed plasma processes attract significant interest for their broad applicability in fields such as nanochemistry $[1,2]$, medicine $[3,4]$, three-dimensional (3D) printing [5], and aerodynamic flow manipulation [6], among many others. Historically, research into pulsed plasma initiation phenomena has focused on gas-phase processes, which as a result are relatively well understood [7,8]. In contrast, electrical discharges in liquids exhibit a complex multiphase environment through which the plasma propagates $[9,10]$, challenging lessons learned from gas-phase discharges. Discharges in liquids are also broadly applicable; therefore, increasing our collective understanding is essential. Recent effort has focused on investigation into liquid-phase breakdown initiation phenomena, of which there are several prevailing hypotheses regarding the dominant breakdown mechanism and currently no conclusive theory [11,12]. It has not yet been proven whether electron avalanching is possible in liquid media; therefore, several groups debate the necessity of preexisting bubbles or dissolved gases for streamer propagation to occur [13-15]. Alternate hypotheses propose the generation of nanopores via electrostriction as a source of low-density regions required for plasma initiation [16]. Still others suggest that field emission from the electrode tip can locally heat the surrounding water, leading to rapid expansion and

\footnotetext{
*zwang@lanl.gov

†staack@tamu.edu
}

Published by the American Physical Society under the terms of the Creative Commons Attribution 4.0 International license. Further distribution of this work must maintain attribution to the author(s) and the published article's title, journal citation, and DOI. a low-density region through which electrons can avalanche [17]. In any case, time-resolved high-resolution imaging of the nanosecond plasma phenomena in question and associated low-density regions can aid in verification/refutation of these candidate initiation mechanisms.

Along with its merit as a phenomenon of primary interest to the fields of plasma physics and engineering, the plasma diagnosed in this work also shows potential for application in the development of new nanosecond imaging systems. This is due to the inherent hypersonic phenomena involved $(29.1 \mathrm{~km} / \mathrm{s})$, as well as low turnaround time between events (less than $1 \mathrm{~s}$ ) compared with other benchmark imaging targets of similar power density (such as exploding wire, which unlike water requires manual reloading). Additionally, plasma initiation can reach high-power densities over short time scales $\left(1 \mathrm{TW} / \mathrm{cm}^{2}\right.$, with $5 \mathrm{~mJ}$ dissipated through $100 \mu \mathrm{m}^{2}$ over $5 \mathrm{~ns}$ ), within two orders of magnitude of power densities used for inertial confinement fusion (ICF) $[18,19]$. An equivalent water plasma setup could therefore be cheaply (total cost $<$ US\$100k) and compactly incorporated as a self-healing benchmark imaging target for diagnostic development at ICF facilities, such as the National Ignition Facility and Laser Mégajoule, as well as at any other facility interested in imaging nanosecond high power-density physical processes [20-22]. In this work we demonstrate the operation of such a device for well-timed interrogation of the water plasma initiation process itself, while also highlighting its value as a repeatable nanosecond imaging target.

\section{PHENOMENA OF INTEREST}

This work focuses on the pulsed plasma shown in Fig. 1, generated using the driving circuit shown in Fig. 2(a). When applying a positive high-voltage pulse to a tungsten electrode 


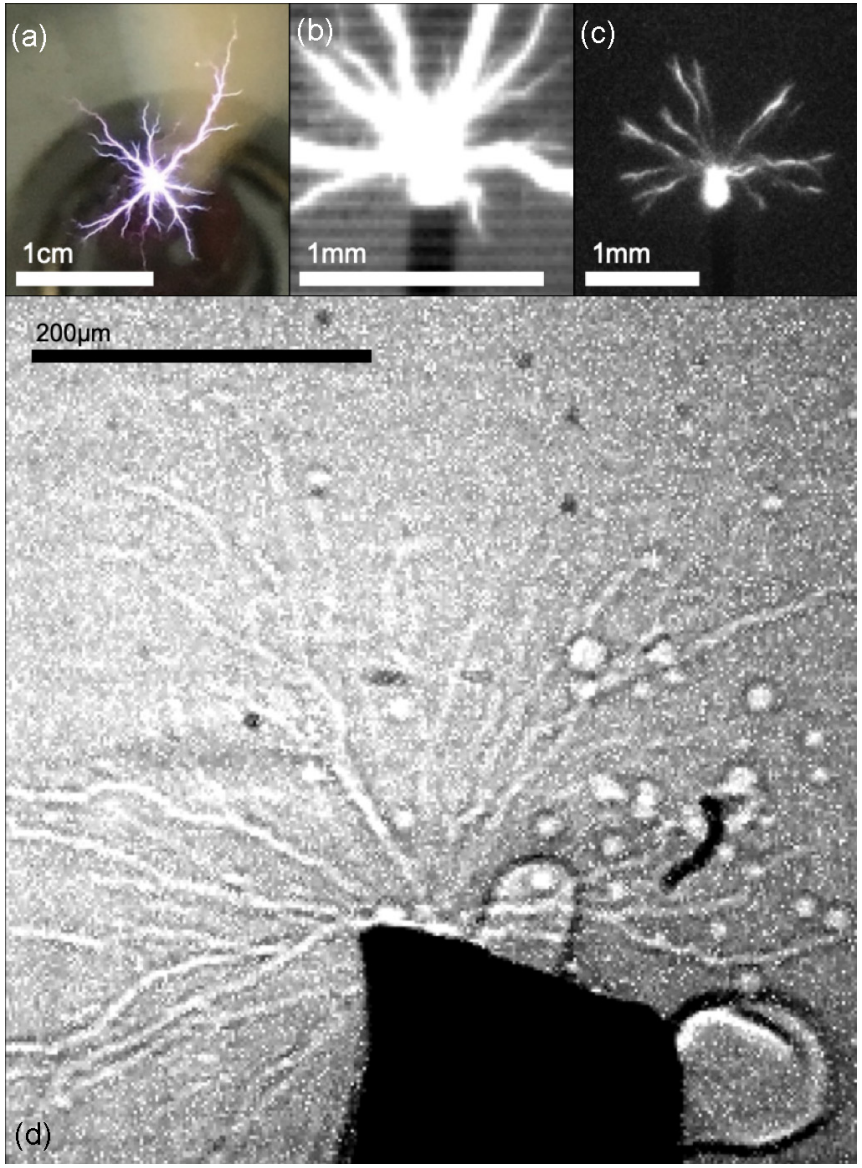

FIG. 1. The pulsed water plasma of interest to this work imaged with four different methods, in order of decreasing exposure time: (a) $67 \mathrm{~ms}$, (b) $2.38 \mu \mathrm{s}$, (c) $10 \mathrm{~ns}$, and (d) $50 \mathrm{ps}$.

submerged in distilled water (filtered to a maximum particle size of $0.2 \mu \mathrm{m}$, with conductivity of roughly $0.5 \mu \mathrm{S} / \mathrm{cm}$ ), a branched-structure plasma forms near sharp-contour regions of the tungsten electrode tip which radiates outward at hypersonic velocities. By triggering an air spark gap switch with a nanosecond laser pulse, repeatable time-resolved diagnostics is possible (similar to setups used in Refs. [13,23]). While a nanosecond-pulsed plasma can occur in a more conductive liquid [24], the branched structures of interest to this work require low conductivity, like that of distilled water or lower. See Supplemental Material, Sec. 1 [25] for a more detailed description of the experimental setups and methods used in this work.

\section{IMAGING RESULTS}

\section{A. Optical Imaging}

Using a high-speed video camera, the plasma of interest was imaged with backlighting at $420 \mathrm{kfps}(2.38 \mu \mathrm{s} /$ frame $)$ and a resolution of $20 \mu \mathrm{m} /$ pixel, shown in Fig. 3(a) and Supplementary Video S1 [25]. Light-emitting plasma channels propagate across the full field of view within the first captured frame of the event, implying a lower bound of $630 \mathrm{~m} / \mathrm{s}$ for propagation speed. Optical emission and plasma energy deposition has ceased by the next frame, and the resulting bubble (a)
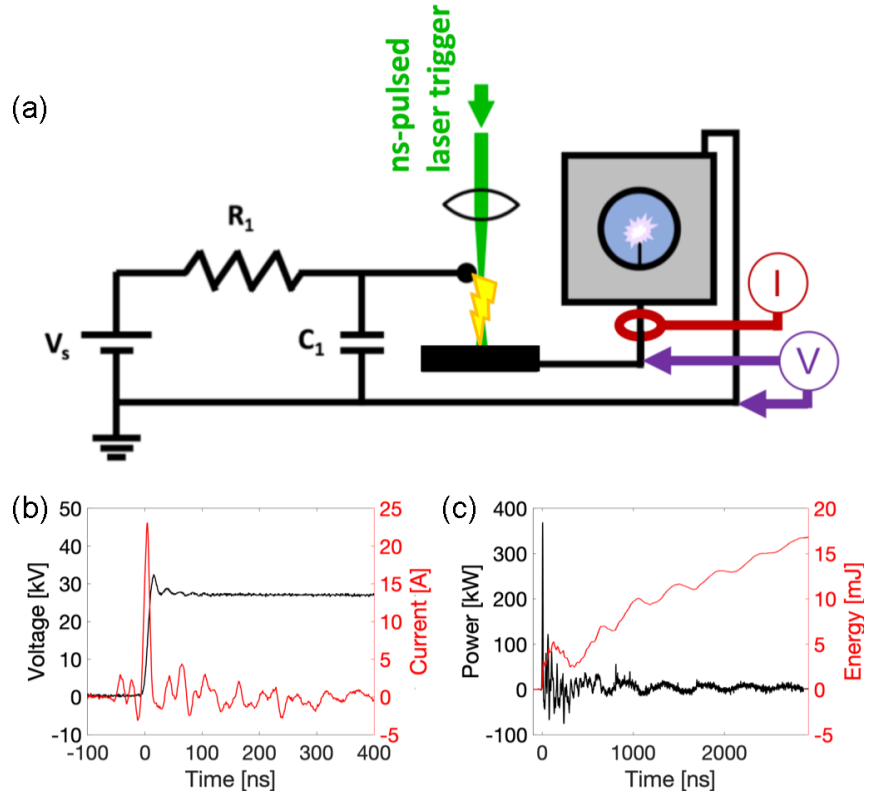

FIG. 2. (a) Diagram of the laser-triggered driving circuit with (b) voltage and current traces for a typical event. (c) Power and energy calculated from direct integration. $R_{1}=20 \mathrm{M} \Omega, C_{1}=1 \mathrm{nF}$, and $V_{b}=+25 \mathrm{kV}$. During this event a total of $242 \mathrm{~mJ}$ was dissipated: $225 \mathrm{~mJ}(93 \%)$ was dissipated by the air spark switch, $5 \mathrm{~mJ}(2 \%)$ contributed to plasma generation, and the remaining $12 \mathrm{~mJ}(5 \%)$ was lost via long-time scale heating and electrolysis. The resulting plasma current pulse had a FWHM of $12 \mathrm{~ns}$ and a peak of $23 \mathrm{~A}$.

begins to evolve and become more spherical over the next $50 \mu \mathrm{s}$.

To better interrogate plasma-time scale processes without requiring ultra-high-frame-rate imaging of a single event, we can take advantage of the fact that this plasma process and experimental setup is well timed. By triggering the event relative to the shutter of a nanosecond-gated camera and logging image timing signals, single event images were sorted according to exposure delay relative to each event. This results in a constructed "video" of water plasma behavior at time scales not achievable via single event high-frame-rate imaging, shown in Fig. 3(b) and Supplemental Video S2 [25]. The plasma (identified by the light-emitting region) initiates near the electrode tip and propagates outward with a hemispherical bush/branching structure over approximately $100 \mathrm{~ns}$, with the longest branches extending almost $4 \mu \mathrm{m}$ away from the electrode before extinguishing. From the naming convention used in Ref. [13], these are exclusively secondary streamers. Outward propagation speed is therefore inferred to be $29.1 \mathrm{~km} / \mathrm{s}$ (Mach 19.7 in water), estimated from the linear trend shown in Figure 3(c). This propagation speed is of the same order as that reported in Ref. [26], as well as in analysis of equivalent $\mathrm{x}$-ray imaging results discussed later in this work [11.7 km/s, from Fig. 5(c)]. This high Mach number suggests that the dominant initiation process is not limited by ambient sound speed, undermining plasma initiation hypotheses which rely on slower processes such as Joule heating or electrolysis. While slower processes may still be significant during longer time scales, they do not appear to drive the channel tip. However, the significant optical emission obscures multiphase 
(a)

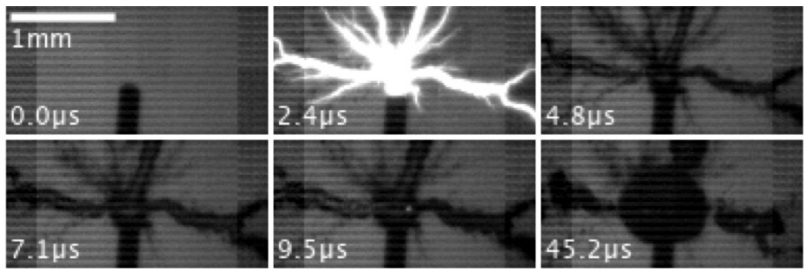

(b)

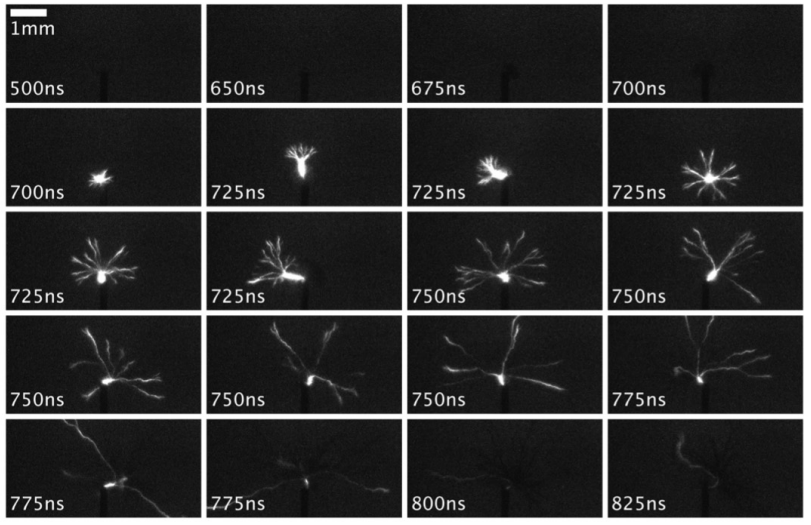

(c)

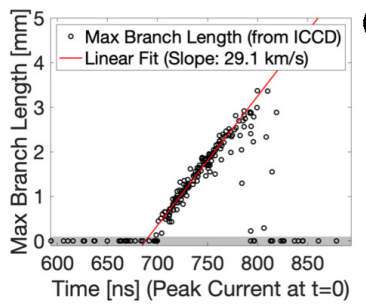

(d)

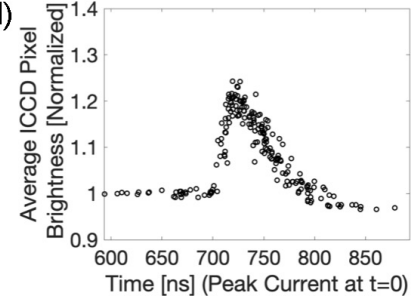

FIG. 3. A series of video frames from high-speed (2.38 $\mu \mathrm{s} /$ frame) backlit photography of a single water discharge event \{(a), Supplemental Video S1 [25]\}. Collection of backlit fastexposure (10 ns) ICCD images from many disparate events, sorted by camera delay relative to time of event as measured by peak PMT signal, with $t=0$ defined to be the average time of peak current $\{(b)$, Supplemental Video S2 [25])\}. Images from (b) were used to generate plots of plasma channel length (c) and average frame brightness (d) vs time.

phenomena needed for more informed discussion of initiation processes. This issue, along with diffraction-limitede resolution at mun scales, prompted our interest in the fast x-ray techniques available at the Advanced Photon Source (APS).

\section{B. X-ray Imaging at APS 32-ID-B}

To integrate our setup into the existing $\mathrm{x}$-ray imaging facilities at the APS 32-ID-B beamline laboratory (see Supplemental Figs. S1 and S2 [25]), several modifications to the water discharge cell and the high-voltage circuit were necessary. Since the x-ray attenuation length of water is $2.2 \mathrm{~cm}$ for the $\mathrm{x}$-ray energies relevant to this work $(24.3 \mathrm{keV})$, a water discharge cell was designed that minimizes the intra-device path length of the x-ray beam to $2 \mathrm{~mm}$.

See Fig. 4 and Supplemental Video S3 [25] for typical $\mathrm{X}$-ray imaging results from separate events, imaged using 24.3-keV x-rays from the APS synchrotron source during a hybrid fill operating mode [27]. The x-ray setup at APS 32-ID-B was configured in a phase-contrast imaging mode, which exploits relative phase delay of the x-ray beam induced by objects on the sample plane with large density gradients $[28,29]$. The gas-liquid boundary of a plasma-induced bubble is therefore easily resolvable in these phase-contrast images with negligible motion blur. See Supplemental Figs. S3-S7 [25] for more x-ray imaging examples of single-events.

Before any quantitative analysis of these $x$-ray images, there are important qualitative observations to be made. In the first postinitiation frame of each imaged event [such as the second frames of Figs. 4(a) and 4(b)], we observe the outward propagation of channellike features at time scales much faster than the interframe period (3.69 $\mu \mathrm{s})$, with presumed dynamic behavior within the first $100 \mathrm{~ns}$ after initiation. Despite saving full initiation-relative delay information for all imaged events and frames, the stochasticity of this plasma processes somewhat hinders insight from multievent comparison.

This phase-contrast imaging method amplifies the effect of discontinuities in density such as surfaces; therefore, we interpret these features to be the liquid-gas interfaces of a lowdensity channel propagating with or near the optical emission front. We also observe correlation in location and propagation direction between these narrow $(\sim 10 \mu \mathrm{m})$ channels and the larger-diameter nonspherical bubbles visible in the subsequent frame $3.69 \mu$ s later, emphasized by the two-frame composite image in Fig. 4(c). Depending on the unequal distribution of energy deposition, the thin plasma branches will either expand into a large diameter bubble (such as the branches at the 8 and 11 o'clock positions in Fig. 4), or will collapse back down to a train of small spherical bubbles (such as the branch at the 9 o'clock position in Fig. 4). This long-lived abnormal bubble shape persists for almost $50 \mu$ s after initiation (see Supplemental Figs. S3-S7 [25]). Based on this, we conclude that significant charge resides near the bubble surface for tens of microseconds after initiation, resulting in long-lived local Coulomb forces after plasma energy deposition. This is consistent with the characteristic charge relaxation time expected for ambient water, $\tau=\sigma / \varepsilon \approx 3.6 \mu \mathrm{s}$. The observed sharpcontour bubbles suggest low local surface tension, caused by the Lippmann effect acting at the locally charged interface. Though the resulting bubble shape is quite striking, processes which dominate at these longer $(\mu \mathrm{s})$ time scales are not necessarily responsible for initiation.

It is also important to acknowledge the plasmaindependent continuous formation of bubbles visible within the region of interest. As previously discussed, the water was sufficiently filtered $(<0.2 \mu \mathrm{m})$, ruling out the possibility of particle contamination. Future work may investigate the effect of degassing on the production of these bubbles; however, in this case we attribute this bubble production to water radiolysis and electrode heating, both induced by high $\mathrm{x}$-ray power $\left(21 \mathrm{~W} / \mathrm{mm}^{2}\right.$ [30]). X-ray-induced bubble production affects the timing of nanosecond plasma initiation processes in water; however, the rapidity of such processes appears to be unaffected during the time scales imaged here. Since these bubbles are present immediately prior to the plasma event both within the volume of interest as well as on the electrode surface, for this set of results we can use their behavior as a rough probe of any forces induced on the fluid. We keep this in mind while recalling the following expression for local 
(a)

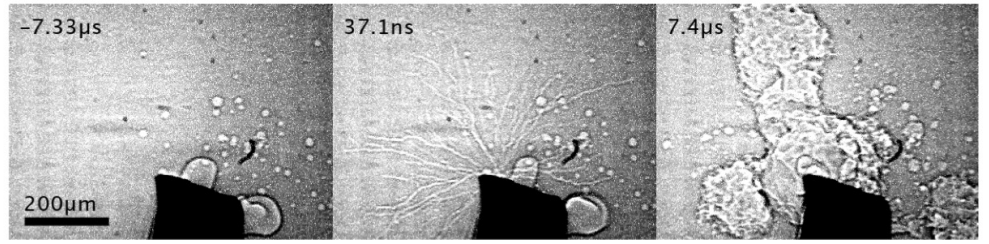

(b)

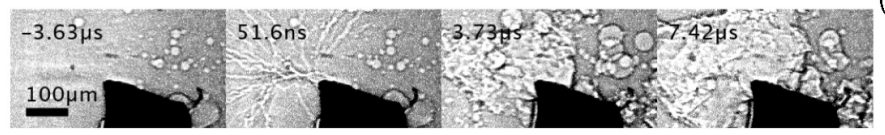

(c)

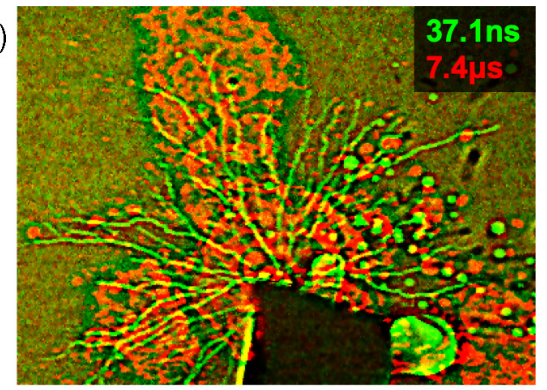

(d)

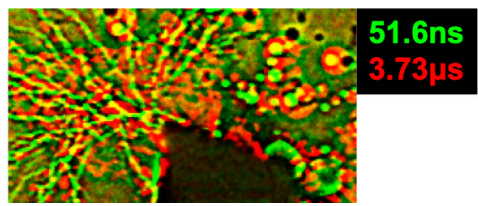

FIG. 4. Frames from ultrafast x-ray imaging videos of two pulsed water plasma events [(a) and (b)], with time stamps annotated relative to peak plasma current as measured through the positive electrode. Frame rates are $136 \mathrm{kfps}$ (a) and $272 \mathrm{kfps}$ (b), or 7.37 and $3.69 \mu \mathrm{s} /$ frame respectively. Resolution is $2 \mu \mathrm{m} /$ pixel. Note the evolution from predischarge to small-diameter long plasma channels to large-diameter cavitation and expansion, as emphasized in (c) and (d) with two-frame composite images from (a) and (b), respectively. See Supplemental Material, Sec. 2 [25] for additional multiframe videos of single events, all of which show correlation in position between the small-diameter channels and larger-diameter bubbles. The event shown in (a) corresponds to Supplemental Video S3 [25].

electrohydrodynamic (EHD) force:

$$
\vec{F}_{\text {EHD }}=q \vec{E}-\frac{\varepsilon_{0}}{2} E^{2} \vec{\nabla} \varepsilon+\frac{\varepsilon_{0}}{2} \vec{\nabla}\left(E^{2} \frac{\partial \varepsilon}{\partial \rho} \rho\right),
$$

(a)

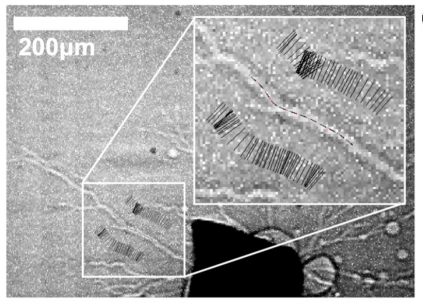

(c)
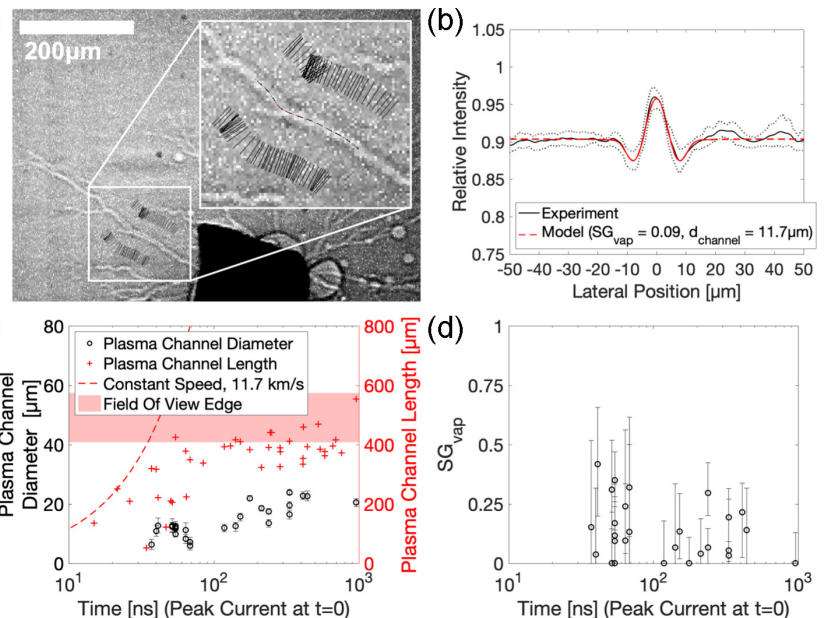

(d)

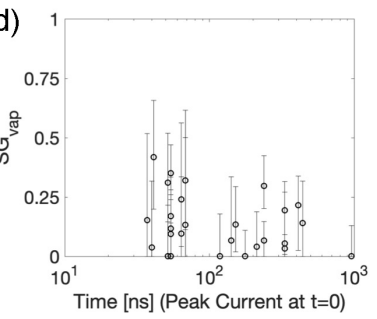

FIG. 5. (a) Illustration of a typical plasma channel cutline interpolation from an $\mathrm{x}$-ray image; (b) typical diffraction model result after optimization relative to the average experimental cutline. Dotted lines represent first and third quartiles of the cutline distribution for each lateral position. For this particular result, the channel diameter and specific gravity were found to be $11.7_{-1.3}^{+1.5} \mu \mathrm{m}$ and $0.09_{-0.09}^{+0.17}$, respectively. Computational results for 27 interpolated experimental cutlines are summarized in (c) $d_{\text {channel }}$ vs $t$ and (d) $S G_{\text {vap }}$ vs. $t$ plots, with time measured relative to water plasma initiation. See Supplemental Figure S9 [25] for a discussion on uncertainty in these plots. Note the general trend that longer times after initiation correlate with larger-diameter plasma channels and lower specific gravities on time scales much faster than the x-ray frame rate. Also included in Fig. 5(c) are the maximum plasma channel lengths (red crosses) taken from each event, the fastest of which implies a channel propagation speed of $11.7 \mathrm{~km} / \mathrm{s}$. where $\varepsilon$ is the permittivity of the liquid, $\varepsilon_{0}$ is the vacuum permittivity, $\rho$ is the mass density of the medium, $\vec{E}$ is the electric field, and $q$ is the free-charge density [16]. The magnitudes of $E, \varepsilon$, and $\rho$ are expected to be either intense or highly nonuniform for our process, and local change density $q$ (and therefore $q \vec{E}$ ) should be relatively high due to Fowler-Nordheim field emission [31] and significant X-ray photoionization. Although this implies a locally intense EHD force which would overwhelm the nanosecond behavior of pre-existing bubbles, we do not see the effect of such a force in these x-ray images; pre-existing bubbles do not react to the nanosecond voltage pulse, only responding after shockwave propagation over several microseconds. The pre-existing bubble behavior is qualitatively quite different than that of the concurrent low-density plasma channels and appears to be independent on initiation time scales, even though some of the smaller pre-existing bubbles are comparable in diameter $(\sim 10 \mu \mathrm{m})$ to the plasma channel and would presumably experience the same electrostrictive forces. We therefore conclude that these experimental results are not consistent with the electrostriction and pre-existing bubble deformation hypotheses for initiation, and that field emission may be aiding in the production of seed electrons (note that x-ray and optical results are qualitatively very similar; therefore, $\mathrm{x}$-ray photoionization is not an significant factor).

\section{X-ray Computational Model}

Unlike a near-field $x$-ray image which can be easily converted to line density, a phase-contrast image requires a more rigorous approach to analyze; in general, a 2D Fresnel-Kirchhoff diffraction integral is required [32]. For this particular case, we can take advantage of both the small scattering angle of the x-ray beam after the sample plane and the cylindrical geometry of a plasma channel to compose a 
simplified integral:

$$
\begin{gathered}
g_{\text {out }}\left(x^{\prime}\right)=\frac{e^{2 \pi i z / \lambda}}{\sqrt{i \lambda z}} \int_{-\infty}^{\infty} g_{\text {in }}(x) e^{\frac{i \pi}{\lambda z}\left(x^{\prime}-x\right)^{2}} d x \\
I_{\text {detector }}\left(x^{\prime}\right)=g_{\text {out }}\left(x^{\prime}\right) g_{\text {out }}^{*}\left(x^{\prime}\right),
\end{gathered}
$$

where $x$ is lateral position relative to the plasma channel at the sample plane and $x^{\prime}$ is lateral position at the detector plane. The complex-valued functions $g_{\text {in }}(x)$ and $g_{\text {out }}\left(x^{\prime}\right)$ describe the electric field at the sample plane and detector plane, respectively. We assume the geometry shown in Supplemental Figure S2 [25], which consists of a simple cylinder of specific gravity $S G_{\text {vap }}$ surrounded by ambient water $\left(S G_{\text {liq }}=1\right)$. The term $g_{\text {in }}(x)$ is therefore defined as follows for a plasma channel of radius $R=\frac{1}{2} d_{\text {channel }}$ in a water cell of width $w_{\text {liq }}$ :

$$
g_{\text {in }}(x)=\left\{\begin{array}{l}
e^{\left[\frac{-2 i \pi}{\lambda} \tilde{n}_{\text {water, liq }} \cdot w_{\text {liq }}\right]},|x|>R \\
e^{\left[\frac{-2 i \pi}{\lambda} \tilde{n}_{\text {water, }, i \mathrm{iq}} \cdot\left(w_{\text {liq }}-2 \sqrt{R^{2}-x^{2}}\right)\right]} \\
\quad \cdot e^{\left[\frac{-2 i \pi}{\lambda} \tilde{n}_{\text {water, vap }} \cdot\left(2 \sqrt{R^{2}-x^{2}}\right)\right]},|x| \leqslant R,
\end{array}\right.
$$

where the complex refractive index $\tilde{n}=1-\delta-i \beta$ is linearly dependent on water density: $\delta_{\text {vap }}=S G_{\text {vap }} \delta_{\text {liq }}$ and $\beta_{\text {vap }}=$ $S G_{\text {vap }} \beta_{\text {liq }}$, with $\delta_{\text {liq }}$ and $\beta_{\text {liq }}$ taken from the literature [33]. Derivation of this model is described in further detail in the Supplemental Material, Sec. 3 [25], which builds off of the general form from Ref. [34] and is equivalent to work by Snigirev on phase-contrast imaging of cylindrical samples [35].

By numerically integrating Eq. (2), we can simulate a phase-contrast x-ray image. Parameters needed for this model include the specific gravity of the region within the plasma channel $S G_{\text {vap }}$, diameter of the channel $d_{\text {channel }}$, as well as three minor parameters which scale and shift the model. To convert the $\mathrm{x}$-ray image into a model-comparable form, we interpolate the image onto a series of cutlines oriented perpendicular to a spline estimation of the plasma channel centerline, forming a cutline intensity distribution for each lateral position. The model is then fit to experimental results to extract estimates of $S G_{\text {vap }}$ and $d_{\text {channel }}$ (see Supplemental Material, Section 3 [25] for details), as shown in Fig. 5.

From this fitted model, $S G_{\text {vap }}$ and $d_{\text {channel }}$ of the plasma channel can be estimated for a particular interpolated cutline, as shown in Fig. 5. The first evident conclusion from these results is that these plasma channels are exclusively low-density phenomena which propagate at speeds comparable to those calculated from the optical emission region (see Fig. 3). This is consistent with initiation hypotheses which require the initial generation of lower-density voids for plasma propagation such as electrostriction and deformation of pre-existing bubbles; however, the aforementioned overall incompatibility of these imaging results with existing initiation hypotheses still stands. It is also important to consider the effect of photonelectron interaction in this environment due to high electron density $\left(\sim 10^{18} \mathrm{~cm}^{-3}\right)$; however, in this case we do not believe that this phenomena has significantly affected these $\mathrm{x}$-ray imaging results. A total of 27 model fits were produced for a variety of selected cutlines at different imaging delays relative to peak current and different distances from the electrode tip \{as shown in Figs. 5(c) and 5(d) and Supplemental Fig. S8 [25]\}, collectively revealing a few major trends in the $\mathrm{x}$-ray data; in particular, larger channel diameters and lower specific gravities occur with increasing time after initiation. Also plotted in Figs. 5(c) and 5(d) are the maximum plasma channel lengths for each frame using a method analogous to that of Fig. 3(c); these results suggest a peak propagation speed of $11.7 \mathrm{~km} / \mathrm{s}$, on the same order as those measured via optical imaging. Low-density regions visible in x-ray therefore evolve at comparable speeds to those of the light-emitting region.

\section{CONCLUSION}

The above work presents an imaging technique which provides insight into the particular mechanism of breakdown for nanosecond-pulsed plasmas in liquids. Using a combination of x-ray and optical methods, we have resolved narrow low-density plasma channels within the streamer head which evolve at speeds $(10-20 \mathrm{~km} / \mathrm{s})$ comparable to those of the light-emitting region. Although the $\mathrm{x}$-ray videos presented here almost exclusively show plasma channels that traverse the full field of view within a single interframe duration, this is still an important result in itself since such rapid plasma channel evolution has not been previously observed due to significant confounding plasma optical emission. To the best of our knowledge, phase-contrast $\mathrm{x}$-ray imaging has not been previously explored as a diagnostic for such plasmas. These results are inconsistent with the electrostriction and bubble deformation hypotheses from the literature, since pre-existing bubbles are almost entirely unaffected by the nanosecond plasma event. This disagreement is currently unresolved; however, the experimental nature of these results gives it precedent over the aforementioned hypotheses which, while rooted in theory, are less evidenced by experiment. Further developments in this direction will no doubt lead to additional insight and possible resolution of this matter.

The superior imaging resolution for this well-timed process and insensitivity to plasma optical emission proves that this technique is useful both as a plasma diagnostic and as a convenient self-healing imaging benchmark; for this work, the overall turnaround time (about $1 \mathrm{~min}$ ) was limited by diagnostic reset time and data logging, which can be minimized to increase acquisition rate. In anticipation of further insight from the combination of these two research avenues, the authors intend to repeat this imaging experiment at the APS in the future with the added experience gained from results reported here. Planned improvements include the use of a sharpened electrode tip (ideally less than $10 \mu \mathrm{m}$ ) for better initiation consistency. Also, note that even with the superior $\mathrm{x}$-ray imaging techniques available at the APS, the frame rate used here still does not completely capture the dynamics of a single event; the plasma initiation phenomena of primary interest occurs over tens of nanoseconds, significantly faster than the $\mathrm{x}$-ray source pulsing frequency. The use of higher $\mathrm{x}$-ray imaging frame rates $(6.67 \mathrm{MHz}$ is expected during future experimental campaigns, compared with $272 \mathrm{kHz}$ in this work) will reveal dynamic processes for single events at time scales on the order of $100 \mathrm{~ns}$, which will further contribute to better understanding of plasma initiation in liquids and the development of faster benchmark imaging targets. 


\section{ACKNOWLEDGMENTS}

Special thanks to the High-Speed Imaging Team at the Los Alamos National Laboratory (LANL) for their collaborative efforts and financial support during these experiments and to the staff at APS 32-ID-B for their time and expertise. LANL work is supported through Triad National Security, LLC
"Triad") by the US Department of Energy (DOE)/NNSA, by the MaRIE Technology Maturation fund, and the $\mathrm{C} 2$ program. This research used resources of the Advanced Photon Source, a DOE Office of Science User Facility operated for the DOE Office of Science by Argonne National Laboratory under Contract No. DE-AC02-06CH11357.
[1] N. Tarasenka, A. Nominé, A. Nevar, M. Nedelko, H. Kabbara, S. Bruyère, J. Ghanbaja, C. Noel, A. Krasilin, G. Zograf, V. Milichko, N. Kulachenkov, S. Makarov, T. Belmonte, and N. Tarasenko, Synergistic Effect of Plasma and Laser Processes in Liquid for Alloyed-Nanoparticle Synthesis, Phys. Rev. Appl. 13, 014021 (2020).

[2] S. Bhattacharyya, D. Staack, E. A. Vitol, R. Singhal, A. Fridman, G. Friedman, and Y. Gogotsi, Localized synthesis of metal nanoparticles using nanoscale corona discharge in aqueous solutions, Adv. Mater. 21, 4039 (2009).

[3] M. S. Hutson and X. Ma, Plasma and Cavitation Dynamics During Pulsed Laser Microsurgery in vivo, Phys. Rev. Lett. 99, 158104 (2007).

[4] G. Fridman, G. Friedman, A. Gutsol, A. B. Shekhter, V. N. Vasilets, and A. Fridman, Applied plasma medicine, Plasma Process Polym. 5, 503 (2008).

[5] C.-C. Shih, M. Burnette, D. Staack, J. Wang, and B. L. Tai, Effects of cold plasma treatment on interlayer bonding strength in fff process, Addit. Manuf. 25, 104 (2019).

[6] J. G. Zheng, Y. D. Cui, Z. J. Zhao, J. Li, and B. C. Khoo, Investigation of airfoil leading edge separation control with nanosecond plasma actuator, Phys. Rev. Fluids 1, 073501 (2016).

[7] Y. Raizer, Gas Discharge Physics (Springer-Verlag, Berlin, Germany, 1991).

[8] A. Piel, Plasma Physics (Springer-Verlag, Berlin, Heidelberg, 2010).

[9] Q. Hu, Z. Zhang, H. Qiu, M. G. Kong, and R. P. Joshi, Physics of nanoporation and water entry driven by a highintensity, ultrashort electrical pulse in the presence of membrane hydrophobic interactions, Phys. Rev. E 87, 032704 (2013).

[10] H.-Y. Chu and H.-K. Lee, Evolution of the Plasma Bubble in a Narrow Gap, Phys. Rev. Lett. 107, 225001 (2011).

[11] P. Bruggeman and C. Leys, Non-thermal plasmas in and in contact with liquids, J. Phys. D 42, 053001 (2009).

[12] J. F. Kolb, R. P. Joshi, S. Xiao, and K. H. Schoenbach, Streamers in water and other dielectric liquids, J. Phys. D 41, 234007 (2008).

[13] W. An, K. Baumung, and H. Bluhm, Underwater streamer propagation analyzed from detailed measurements of pressure release, J. Appl. Phys. 101, 053302 (2007).

[14] J.-H. Shiu and H.-Y. Chu, Finger evolution of a gas bubble driven by atmospheric pressure plasma, Phys. Rev. E 94, 063201 (2016).

[15] A. Starikovskiy, Y. Yang, Y. I. Cho, and A. Fridman, Nonequilibrium plasma in liquid water: Dynamics of generation and quenching, Plasma Sources Sci. Technol. 20, 024003 (2011).

[16] Y. Seepersad, A. Fridman, and D. Dobrynin, Anode initiated impulse breakdown in water: The dependence on pulse rise time for nanosecond and sub-nanosecond pulses and initiation mechanism based on electrostriction, J. Phys. D 48, 424012 (2015).

[17] H. M. Jones and E. E. Kunhardt, Development of pulsed dielectric breakdown in liquids, J. Phys. D 28, 178 (1995).

[18] J. Nuckolls, Feasibility of inertial-confinement fusion, Phys Today 35(9), 24 (1982).

[19] R. Betti and O. A. Hurricane, Inertial-confinement fusion with lasers, Nat. Phys. 12, 435 (2016).

[20] C. J. Cerjan, L. Bernstein, L. B. Hopkins, R. M. Bionta, D. L. Bleuel, J. A. Caggiano, W. S. Cassata, C. R. Brune, D. Fittinghoff, J. Frenje, M. Gatu-Johnson, N. Gharibyan, G. Grim, C. Hagmann, A. Hamza, R. Hatarik, E. P. Hartouni, E. A. Henry, H. Herrmann, N. Izumi et al., Dynamic high energy density plasma environments at the national ignition facility for nuclear science research, J. Phys. G: Nucl. Part. Phys. 45 , 033003 (2018).

[21] S. R. Nagel, A. C. Carpenter, J. Park, M. S. Dayton, P. M Bell, D. K. Bradley, B. T. Funsten, B. W. Hatch, S. Heerey, J. M. Hill, J. P. Holder, E. R. Hurd, C. C. Macaraeg, P. B. Patel, R. B. Petre, K. Piston, C. A. Trosseille, K. Engelhorn, T. J. Hilsabeck, T. M. Chung et al., The dilation aided singleline-of-sight $\mathrm{x}$-ray camera for the national ignition facility: Characterization and fielding, Rev. Sci. Instrum. 89, 10 G125 (2018).

[22] B. J. Little, Characterization of a picosecond gated optical intensifier, Rev. Sci. Instrum. 89, 10E117 (2018).

[23] N. C. Lopes, G. Figueira, L. O. Silva, J. M. Dias, R. Fonseca, L. Cardoso, C. Russo, C. Carias, G. Mendes, J. Vieira, and J. T. Mendonça, Plasma channels produced by a laser-triggered highvoltage discharge, Phys. Rev. E 68, 035402(R) (2003).

[24] P. Xiao and D. Staack, Microbubble generation by microplasma in water, J. Phys. D 47, 355203 (2014).

[25] See Supplemental Material at http://link.aps.org/supplemental/ 10.1103/PhysRevResearch.3.L022021 for detail on the x-ray imaging experimental setup, more $\mathrm{x}$-ray imaging results from selected pulsed plasma events in water, derivation of the computational model, discussion of uncertainty analysis, and Supplemental video files.

[26] P. H. Ceccato, O. Guaitella, M. R. L. Gloahec, and A. Rousseau, Time-resolved nanosecond imaging of the propagation of a corona-like plasma discharge in water at positive applied voltage polarity, J. Phys. D 43, 175202 (2010).

[27] M. Borland, G. Decker, L. Emery, W. Guo, K. Harkay, V. Sajaev, and C.-Y. Yao, APS storage ring parameters, https: //ops.aps.anl.gov/SRparameters, Sept. 2010.

[28] P. C. Diemoz, M. Endrizzi, C. E. Zapata, Z. D. Pešić, C. Rau, A. Bravin, I. K. Robinson, and A. Olivo, X-Ray Phase-Contrast Imaging with Nanoradian Angular Resolution, Phys. Rev. Lett. 110, 138105 (2013). 
[29] M. Endrizzi, F. A. Vittoria, L. Rigon, D. Dreossi, F. Iacoviello, P. R. Shearing, and A. Olivo, X-Ray Phase-Contrast Radiography and Tomography With a Multiaperture Analyzer, Phys. Rev. Lett. 118, 243902 (2017).

[30] M. S. del Rio and R. J. Dejus, Status of XOP v2.4: recent developments of the $\mathrm{x}$-ray optics software toolkit, SPIE Proc. 8141, 814115 (2011).

[31] S.-D. Liang, Quantum Tunneling and Field Electron Emission Theories (World Scientific, Singapore, 2014).

[32] F. L. Pedrotti, S. J, L. M. Pedrotti, and L. S. Pedrotti, Introduction to Optics, 3rd ed. (Pearson Prentice Hall, New Jersey, 2007).
[33] B. L. Henke, E. M. Gullikson, and J. C. Davis, X-ray interactions: photoabsorption, scattering, transmission, and reflection at $\mathrm{E}=50-30000 \mathrm{eV}, \mathrm{Z}=1-92$, At. Data Nucl. Data Tables 54, 181 (1993).

[34] G. Barbastathis, C. Sheppard, and S. B. Oh, 2.71 Optics, Lecture 15, Massachusetts Institute of Technology: MIT OpenCourseWare, https://ocw.mit.edu, License: Creative Commons BY-NC-SA., Spring 2009.

[35] A. Snigirev, I. Snigireva, V. Kohn, S. Kuznetsov, and I. Schelokov, On the possibilities of X-ray phase contrast microimaging by coherent high-energy synchrotron radiation, Rev. Sci. Instrum. 66, 5486 (1995). 